\title{
Pharyngeal timing and particle transport defects in Caenorhabditis elegans feeding mutants
}

Isaac Ravi Brenner ${ }^{1}$, David M. Raizen², and Christopher Fang-Yen ${ }^{2,3,{ }^{*}}$

${ }^{1}$ Department of Bioengineering, School of Engineering and Applied Science, University of Pennsylvania, Philadelphia PA, 19104

2Department of Neurology, Perelman School of Medicine, University of Pennsylvania, Philadelphia PA, 19104

${ }^{3}$ Department of Neuroscience, Perelman School of Medicine, University of Pennsylvania, Philadelphia PA, 19104

*Corresponding Author. Email: fangyen@seas.upenn.edu Mailing address: 210 S 33 ${ }^{\text {rd }}$ St., Skirkanich Hall 240, Philadelphia, PA 19104

Abstract: The nematode $C$. elegans uses rhythmic muscle contractions and relaxations called pumps to filter, transport, and crush food particles. A number of feeding mutants have been identified, including those with slow pharyngeal pumping rate, weak muscle contraction, defective muscle relaxation, and defective grinding of bacteria. Many aspects of these pharyngeal behavioral defects and how they affect pharyngeal function are not well understood. For example, the behavioral deficits underlying inefficient particle transport in 'slippery' mutants have been unclear. Here we use high speed video microscopy to describe pharyngeal pumping behaviors and particle transport in wild-type animals and in feeding mutants. Different 'slippery' mutants exhibit distinct defects including weak isthmus contraction, failure to trap particles in the anterior 
24 isthmus, and abnormal timing of contraction and relaxation in pharyngeal compartments. Our results show that multiple deficits in pharyngeal timing or contraction can cause defects in particle transport.

Abbreviated title: Timing and transport defects in C. elegans feeding mutants

\section{Introduction}

The nematode $C$. elegans consumes food bacteria using movements of the pharynx, a neuromuscular feeding organ that connects to the buccal cavity and to the intestine. The pharynx displays two behaviors: pharyngeal pumping and isthmus peristalsis. open the pharyngeal lumen; during relaxation the lumen closes. Isthmus peristalsis is an anterior-to-posterior contraction in the isthmus that occurs after a fraction of pumps (Avery and Horvitz, 1987). Together, these movements filter food particles from

41 surrounding fluids, transports the particles posteriorly, and crushes them before they 42 enter the intestine.

44 The pharynx is divided into several compartments including the anterior and posterior

45 (terminal) bulbs. Detailed studies of pharyngeal pumping have revealed differences in

46 timing of contractions and relaxations in these compartments (Avery, 1993a, 1993b; 
47 Fang-Yen et al., 2009). The corpus, which extends from the buccal cavity to the

48 anterior bulb, is the first to contract, drawing particles from the buccal cavity into the

49 pharyngeal lumen. Contraction of the isthmus and then the terminal bulb follow

50 contraction of the corpus with short delays. Pharyngeal relaxation of the various

51 compartments proceeds in the same order as their contraction but several times more

52 rapidly (Fang-Yen 2009).

54 The sequence of relaxations in pharyngeal compartments is closely tied to the

55 mechanism of particle trapping. The anterior tip of the corpus relaxes slightly before the

56 rest of the corpus; this allows food particles to become trapped at the anterior corpus.

57 Similarly, relaxation of the metacorpus before the anterior isthmus allows food particles

58 to become trapped at the anterior isthmus. At each of the two trap sites (anterior corpus

59 and anterior isthmus), earlier relaxation of the structures immediately anterior to the trap

60 prevents anteriorward transport of particles while fluids are expelled (Fang-Yen et al.,

61 2009). Under some conditions such as violet light exposure, the trap at the anterior

62 procorpus remains contracted, causing particles to be ejected from the anterior pharynx

63 rather than trapped at the end of the pump (Bhatla et al., 2015; Sando et al., 2021). 
64 Particles trapped in the anterior isthmus are transported to the pharyngeal grinder via

65 isthmus peristalsis.

67 A number of 'eat' mutants with defective feeding behavior were identified by Avery

68 (Avery, 1993a).

69 Feeding phenotypes included slow pumping, defective timing of relaxation, and a

70 "slippery pharynx" phenotype characterized by inefficient transport of particles. These

71 feeding mutants were described using video recordings at 60 frames per second (fps), a

72 frame rate insufficient to resolve the rapid movements of the pharynx and food particles

73 during muscle relaxation. As a result, aspects of pharyngeal behavior in feeding

74 mutants have remained unclear. In particular, the mechanisms by which 'slippery'

75 mutants are defective in anterior-to-posterior particle transport are poorly understood.

77 Here, we revisit the analysis of feeding mutants using high speed video microscopy at

781000 fps. We imaged worms feeding on bacteria and on polystyrene beads. By

79 tracking the motions of pharyngeal components and particles within the pharyngeal

80 lumen, we quantified the timing of contraction and relaxation, motion of particles in the

81 pharyngeal lumen, and efficiency by which particles were transported and trapped. We

82 also measured the pumping rate and the frequency of posterior isthmus peristalsis, by

83 which particles trapped in the anterior isthmus are transported to the terminal bulb.

84 Different slippery mutants showed a variety of behavioral defects, including weak

85 isthmus contraction, failure to trap particles in the anterior isthmus, and abnormal timing 
86 of contractions and relaxations in different pharyngeal compartments. We also describe

87 behavioral differences in slow-feeding and relaxation-defective mutants.

\section{Results}

The class of slippery mutants were labeled as such by Avery because in these animals,

bacteria were observed to slip toward the anterior instead of being trapped during

93 pharyngeal relaxation (Avery, 1993a). We asked what differences in pharyngeal

94 behavior relative to wild-type animals led to the slippery phenotype.

We first used our assay to describe wild-type pharyngeal behavior. In wild-type animals, pump timing and particle movement were consistent with previous findings (Fang-Yen et al., 2009). Each pump began with the onset of contraction of the corpus, followed $28.5 \pm 14.8 \mathrm{~ms}$ (mean $\pm \mathrm{SD}$ ) later by onset of contraction of the anterior isthmus, followed $9.6 \pm 11.7 \mathrm{~ms}$ (mean $\pm \mathrm{SD}$ ) later by onset of contraction of the terminal bulb (Fig, 1b, Video S1, Supplemental Table A3). Onset of relaxation occurred in the same order but faster, beginning with the corpus, followed $13.4 \pm 9.9$ ms later by

103 the anterior isthmus, and then $6.4 \pm 10.7 \mathrm{~ms}$ later by the terminal bulb (Fig. 1b). The pumping rate was $4.4 \mathrm{~Hz} \pm 0.48$ (mean $\pm \mathrm{SD}$ ). Isthmus peristalsis occurred after $22.6 \%$ of pumps, consistent with prior studies (Avery, 1993a; Song and Avery, 2012).

To assay the efficiency of particle transport, we reviewed video sequences to manually track trajectories of particles as they moved through the pharynx (see Methods). We 
109 found that pharynxes in wild-type animals transported particles efficiently: 79.5\% (35 out

110 of 44 ) of tracked particles in the corpus at the beginning of contraction reached the

111 anterior isthmus and were trapped there at the end of the relaxation phase

112 (Supplemental Table A4).

\section{Various behavioral defects contribute to inefficient transport in slippery mutants}

We analyzed four slippery mutants: eat-10, eat-13, eat-15, and eat-17. By reviewing video recordings, we examined the patterns and timing of contraction and relaxation of pharyngeal compartments in each mutant. We also analyzed transport of particles 122 (bacteria and/or polystyrene beads). In wild-type animals, robust contraction of the anterior isthmus causes particles in the corpus to be drawn into the isthmus (Fig. 1a,b) where they can be trapped. By contrast, in eat-10 mutants the anterior isthmus frequently either failed to contract (18/49 pumps, $36 \%)$ or contracted weakly (14/49 pumps, 29\%). Neither weak nor absent contractions

128 were ever observed in wild-type worms. During weak contractions in eat-10 worms,

129 there was very limited (<20\% of normal) opening of the pharyngeal lumen (Fig. 1c,

130 Video S2 worms 3-4). In cases of weak or absent anterior isthmus contraction, particles 131 were not drawn into the anterior isthmus (Fig. 1c, Video S2, Worm 3-4). As a result, in 
132 eat-10 mutants, only $33 \%$ (16 out of 49 ) of the particles starting in the corpus at the

133 beginning of a pump became trapped in the anterior isthmus at the end of the pump. In

134 comparison, in wild-type animals, $79.5 \%$ moved from the corpus to isthmus at the end

135 of the pump (Fig. 4). This finding is consistent with the original description of slippery

136 pumping in which many particles reach the metacorpus at the peak of contraction but

137 then return to the anterior procorpus during relaxation (Avery 1993a). 


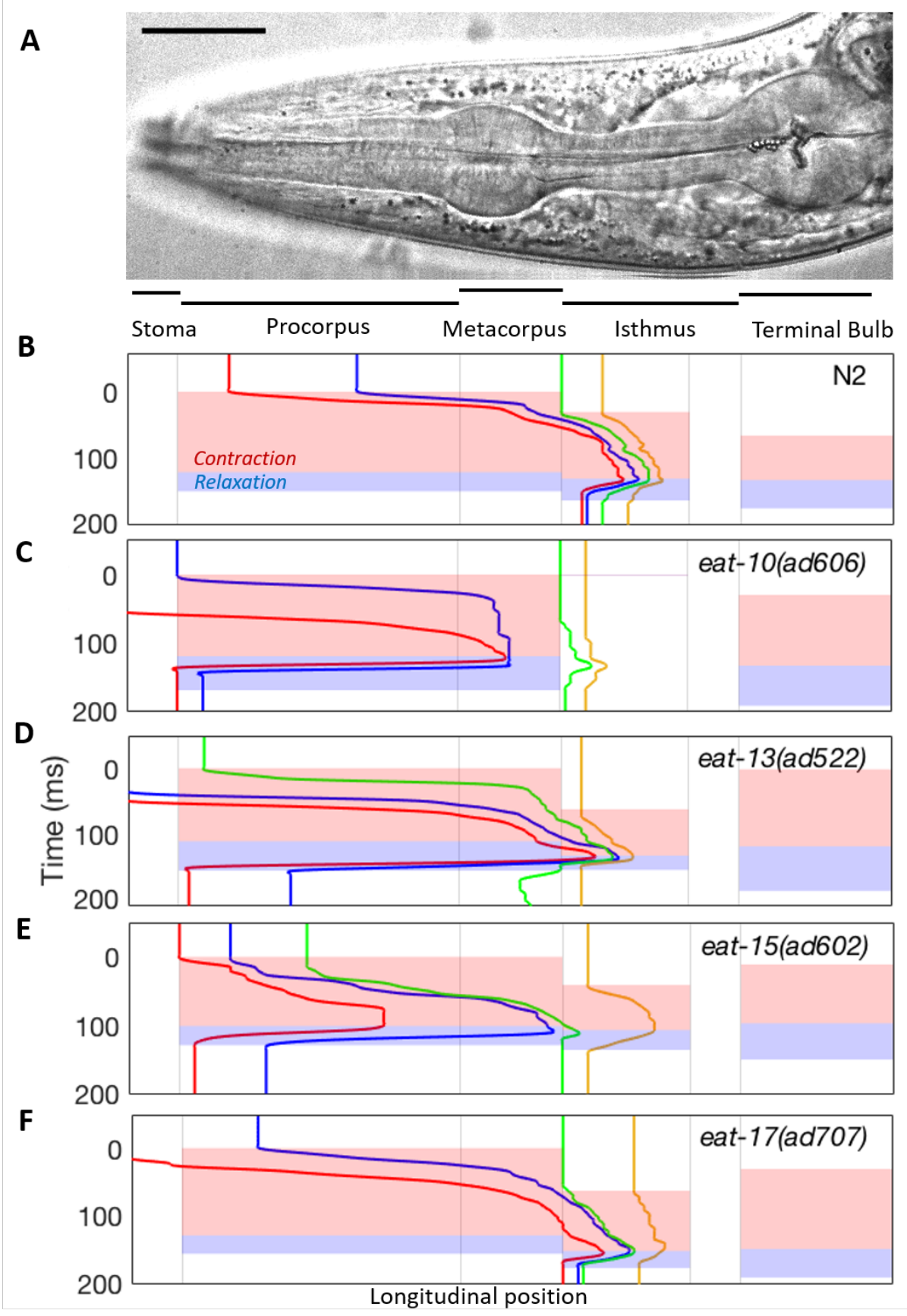

Figure 1: Particle tracking and pharyngeal subcomponent timing of wild type

140 worms and slippery mutants. (A) The pharynx and pharyngeal compartments in a

141 wild type animal. Scale bar: $25 \mu m$. (B-F) Pharyngeal muscle dynamics and particle

142 tracking data from high-speed video microscopy of a single representative pump cycle

143 from wild type (N2) and mutant worms. Colored traces indicate trajectories of four 
144 particles. Colored areas show timings of contraction and relaxation of the corpus,

145 anterior isthmus, and terminal bulb, with red representing contraction and blue

146 representing relaxation. The $x$ axis corresponds to the longitudinal positions shown in

147 (A). For eat-10, no isthmus contraction or relaxation was visible during this pump

148 (Panel C, Supplemental Video S2 worm 4). Details of particle tracking data are given in

149 Supplemental Table A4.

151 As in eat-10 mutants, we found in eat-15 worms a low $(20 / 42=47.6 \%)$ rate of particle

152 transport from the corpus to isthmus compared to wild type (Fig. 4). In some pumps,

153 isthmus contraction was weak (Video S4 worm 3). Particle tracking showed that

154 particles initially located in the corpus often did not enter the isthmus, instead stalling

155 upon reaching the metacorpus (Fig. 1e). In some pumps, the particles moved back and

156 forth along the lumen during relaxation, perhaps due to influence from movements in

157 the terminal bulb (e.g. Video S4 worm 4).

159 In eat-13 mutants, efficiency of transport from the corpus to isthmus $(24 / 38=63 \%)$ was 160 only slightly lower than that of wild-type animals (79.5\%). Pharynxes in eat-13 mutants

161 appeared physically distorted: the ratio of the isthmus width and the terminal bulb width

162 to the length of the pharynx in eat-13 mutants was greater than that ratio in wild-type

163 worms (Video S3, Fig. 2a-e). This difference in morphology is unlikely to be explained

164 by a nutritional defect alone since eat-2 mutants, which have a defect in cholinergic

165 excitation of the pharynx and therefore pump slowly (McKay et al., 2004; Raizen, Lee,

166 and Avery, 1995), had a ratio no different from that of wild-type worms. 

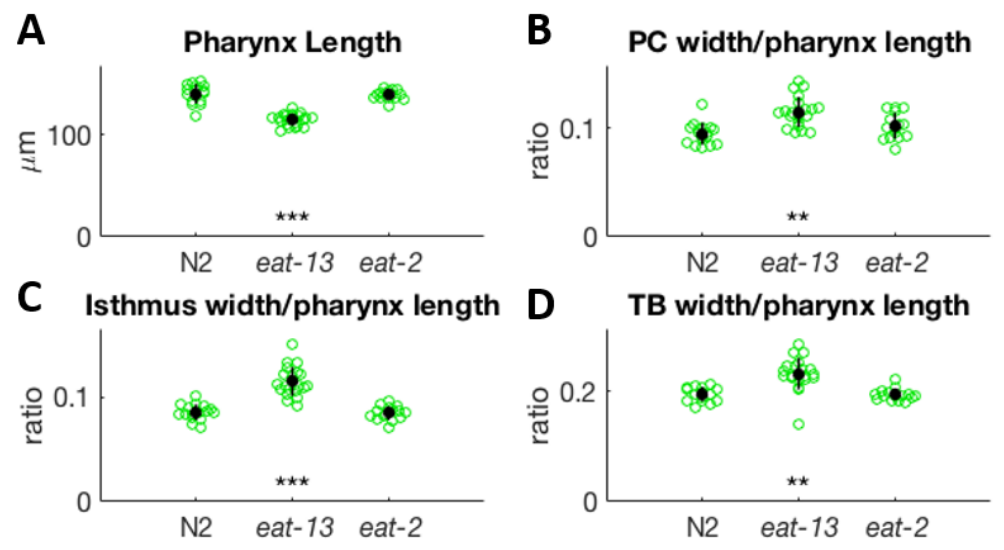

E eat-13(ad522)

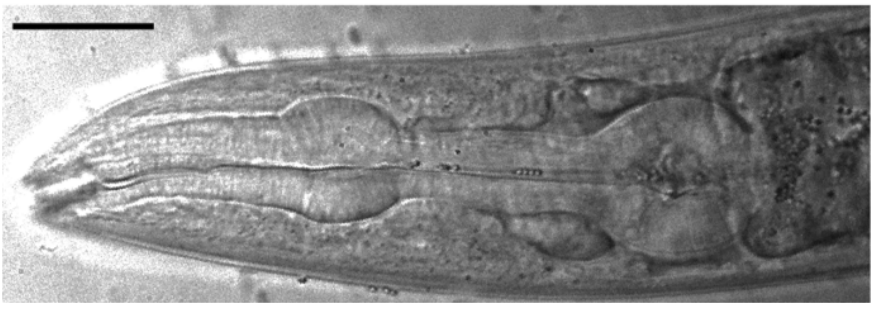

F eat-17(ad707)

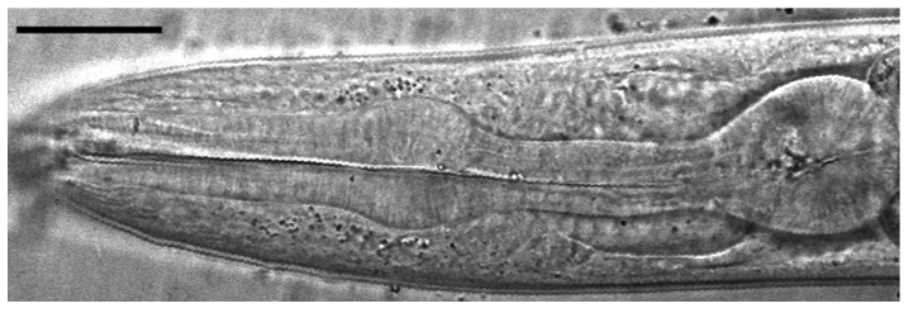

171 Figure 2: Pharynxes of eat-13 animals are distorted. (A) Pharynx length for N2, eat-13,

172 and eat-2 animals. (B) Ratio of procorpus width to pharynx length. (C) Ratio of isthmus

173 width to pharynx length. (D). Ratio of TB width to pharynx length. Significance threshold

174 from t-test: ${ }^{*}$ indicates $p<0.05,{ }^{* *}$ indicates $p<10^{-5},{ }^{* * *}$ indicates $p<10^{-8}$. Note that

175 the eat-13 pharynx is generally shorter and wider than N2. See Supplemental Table A5 
176 for detailed measurements. (E) Deformed eat-13 pharynx. (F) Pharynx of an eat-17 worm has a deformed grinder. See also Video S5. Scale bar: $25 \mu \mathrm{m}$ for both images.

The gene eat-17 encodes a GTPase-activating protein required for pharyngeal cuticle structure (Straud et al., 2013). In eat-17 mutants, we observed a high percentage of particles failing to make posterior progress along the pharyngeal lumen (29\% compared to $14 \%$ in wild-type, Supplemental Table A4). However, unlike eat-13 mutants, eat-17 mutants were highly efficient in corpus-to-isthmus transport, suggesting that most of the transport inefficiency in eat-17 mutants was occurring in the isthmus. The grinder in the terminal bulb of eat-17 mutant pharynxes appeared deformed (Video S5, Fig. 2f), as previously reported (Straud et al., 2013). Overall, eat-17 mutants were able to transport particles from the corpus to the isthmus efficiently, but had defective transport within the

188 isthmus (Video S5).

\section{Slippery mutants display altered isthmus and terminal bulb contraction timings}

In wild-type worms, the onset of contraction of the terminal bulb nearly always occurred after onset of contraction in the isthmus, and the onset of relaxation of the terminal bulb occurred predominantly after onset of relaxation in the isthmus (Fig. 3, Supplemental Table A3). In eat-10, eat-13, and eat-15 animals, the terminal bulb began contracting earlier on average than the isthmus (Fig 3a). Similarly, on average, the terminal bulb 
198 also began relaxation before the isthmus (Fig. 3b), although the difference was smaller

199 than that of the contraction delay.

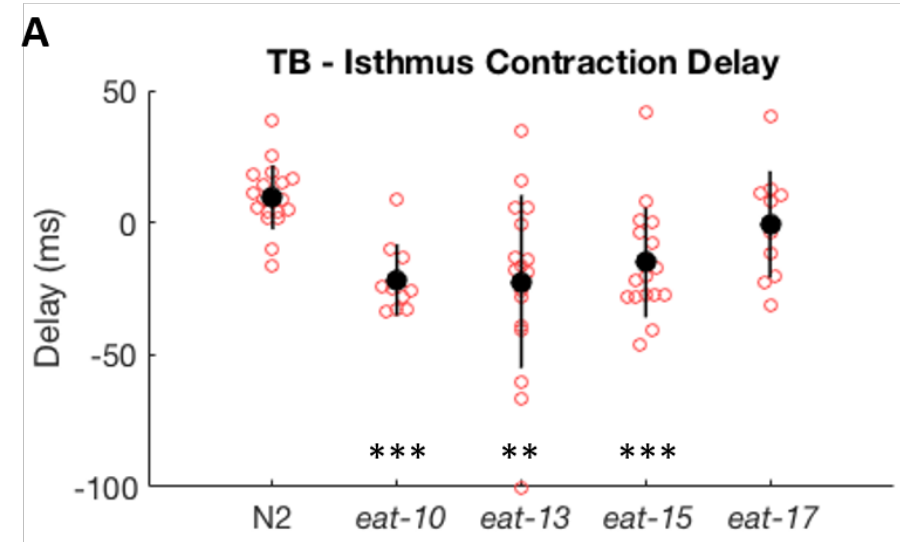

B

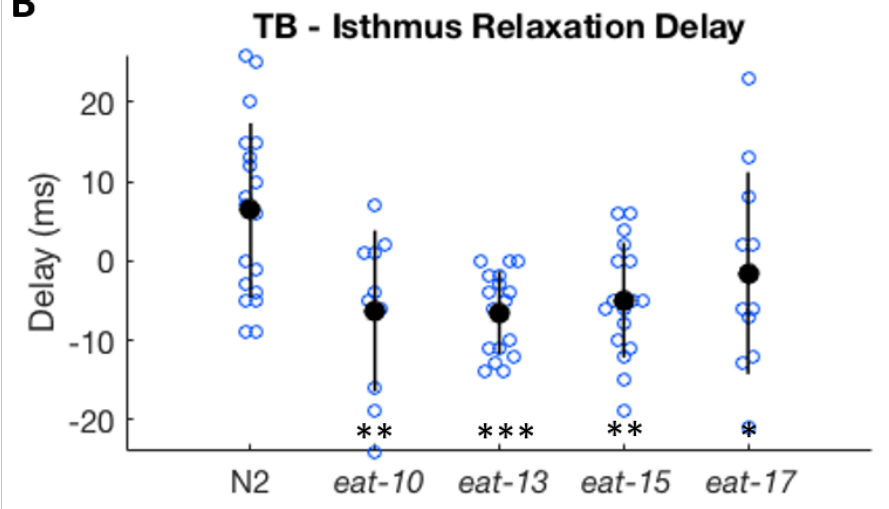

201 Figure 3: Delay between the onset of $(A)$ contraction and $(B)$ relaxation of the terminal

202 blub (TB) relative to the isthmus in wild-type worms and slippery mutants. Negative

203 delay indicates that terminal bulb contracted/relaxed prior to isthmus. Each point

204 represents a single pump. For each genotype, data is from 1 pump from each of $\mathrm{N}=$

205 10-19 animals (See Supplemental Table A3). Error bars indicate +/- SEM. Results of

206 two-tailed Student's t-test comparing each strain to N2; ${ }^{*}$ indicates $p<0.05$, ${ }^{* *}$ indicates

$207 \mathrm{p}<0.005,{ }^{* * *}$ indicates $\mathrm{p}<0.0005$. 


\section{Relaxation defective mutants show defective transport}

The pharynxes of loss-of-function mutants eat- 6 fail to relax completely during the pump cycle (Avery, 1993a). The gene eat-6 encodes a sodium/potassium ATPase, a pump required to maintain the resting membrane potential (Davis et al., 1995). In eat-6 mutants, we observed that the terminal bulb only occasionally came back to its fully relaxed position, usually appearing to only partially relax (Video S6). In addition to this failure of the terminal bulb to fully relax, the relaxation phases were proportionally longer in eat- 6 mutants than in the wild type. Corpus $(p=0.001)$ and isthmus $(p=0.02)$ contraction phases were significantly shorter in eat-6 after normalizing for pump period, indicating that relaxation made up a greater percentage of the overall pump. Like eat-10 animals, eat- 6 worms frequently showed weak isthmus contractions, precluding effective transport (Fig. 5c, Video S6 worms 2-4). This resulted in a lower rate of corpus to isthmus transport (23.2\% compared to $79.5 \%$ in N2; Fig. 4).

Like eat-6 mutants, gain-of-function mutants of egl-19 (an L-type voltage-gated calcium channel (Lee et al 1997) fail to relax completely during the pump cycle (Avery, 1993a). In an egl-19 gain-of-function mutant, the most notable behavior was occasional (3/46 pumps) isthmus and terminal bulb "yawns" in which those parts of the pharynx would remain contracted while the corpus went through its normal pump cycle (Video S7 worm 1-2). These terminal bulb contractions would span two pumps of the corpus. Even when the contraction ended prior to the next pump (i.e., no discernable "yawn" occurred), the isthmus and terminal bulb remained contracted longer than observed in wild-type worms (Supplemental Table A2). Consistent with this, we found a longer delay between onset 
233 of corpus relaxation, and that of the isthmus, and subsequently the terminal bulb

234 (Supplemental Table A3). We observed a lower rate of corpus to isthmus transport

$235(18 / 40,45 \%)$, as well as an increase in particles ending in the metacorpus $(21 \%$

236 compared to $0 \%$ in wild type (Fig. 4, Supplemental Table A4).

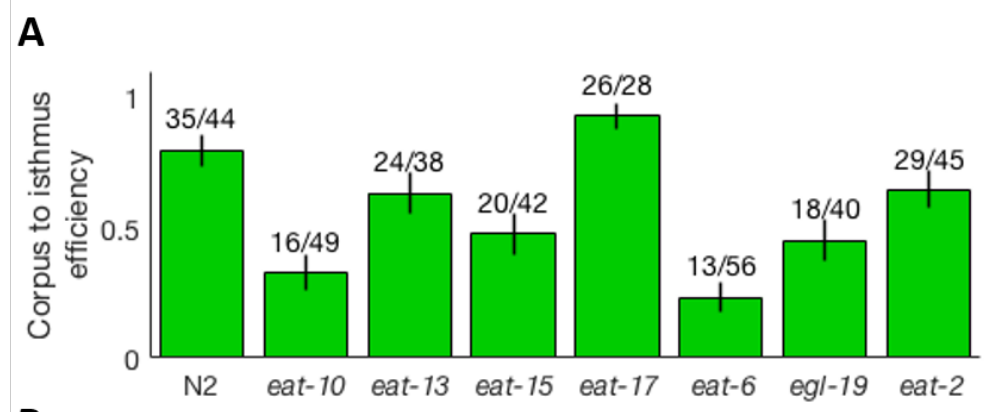

B

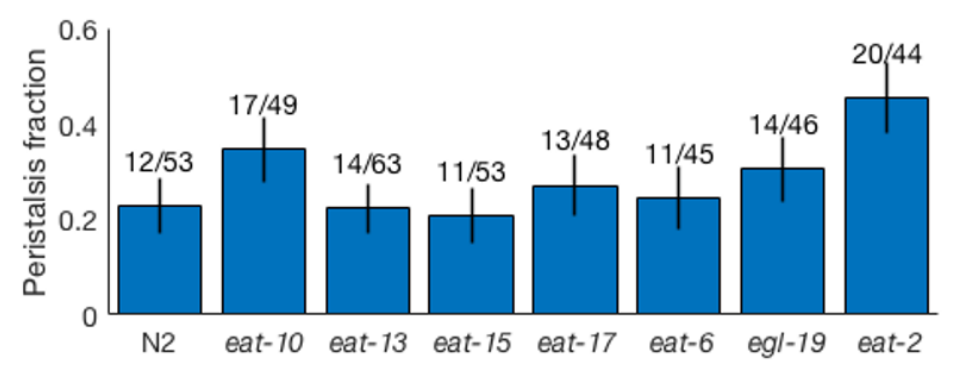

C

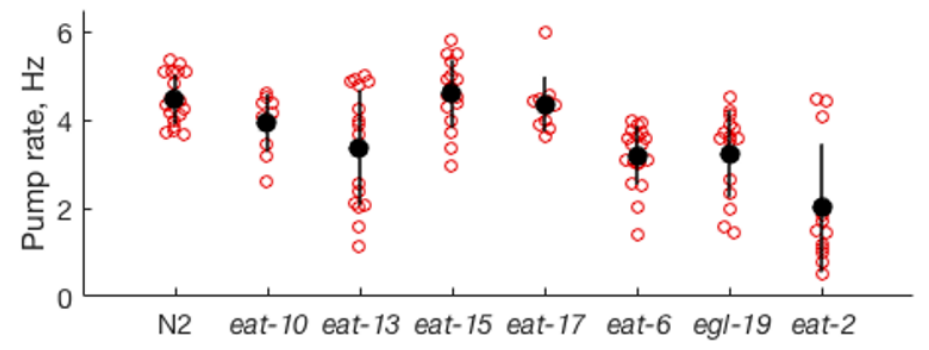

Figure 4: (A) Particle transport efficiency, defined as the fraction of particles starting in

239 the corpus that are transported to the isthmus during a pump. Error bars show \pm

240 standard deviation assuming a binomial distribution. (B) Fraction of pumps that were

241 followed by isthmus peristalsis. Error bars show standard deviation assuming a 
242 binomial distribution. (C) Pump rate, with black bars indicating mean +/- SD. Additional

243 data can be found in Supplemental Tables A1 and A4.

A

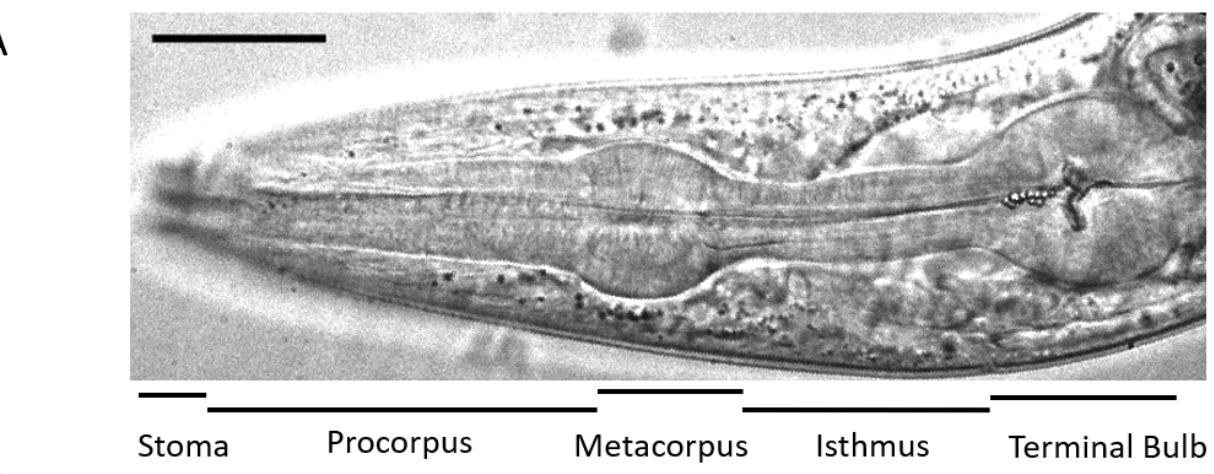

B

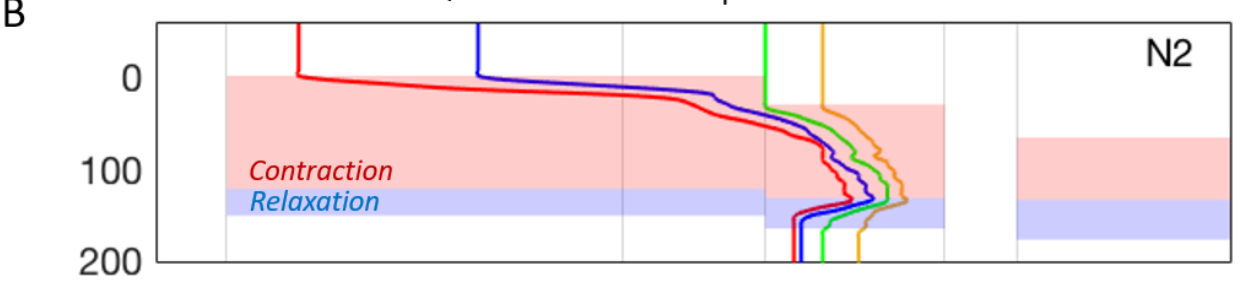

$\mathrm{C}$

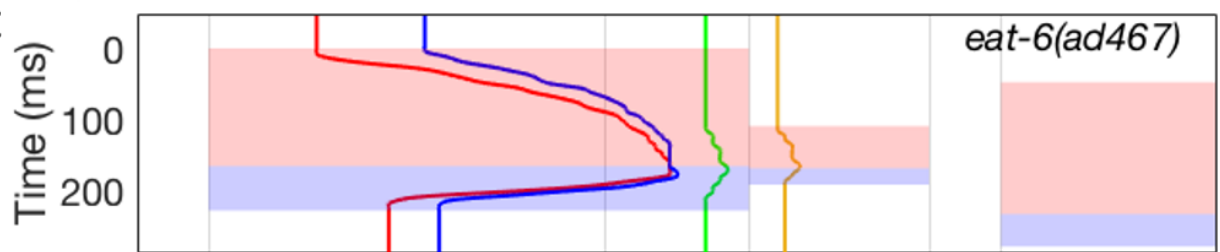

D

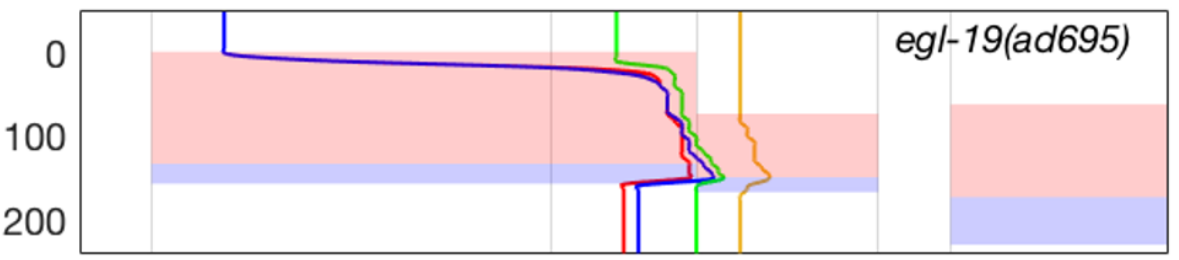

$\mathrm{E}$

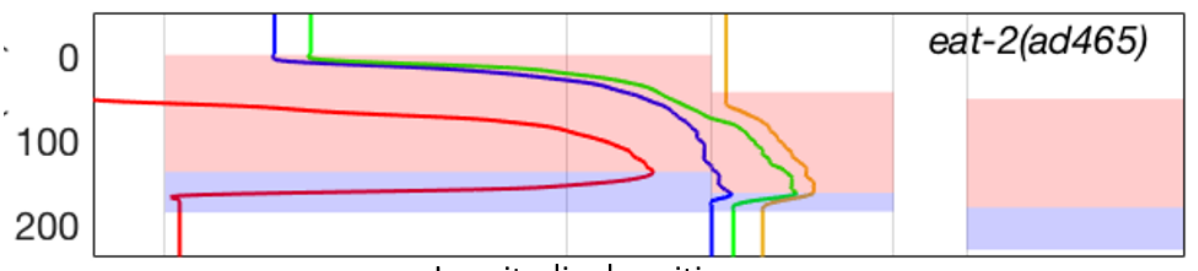

Longitudinal position

Figure 5. Particle tracking and pharyngeal subcomponent timing of N2, eat-6, egl-

248 19, and eat-2 mutants. (A-E) Representative particle trajectories and

249 contraction/relaxation timings, as in Fig. 1. 


\section{Slow pumping mutant eat-2 shows normal pharyngeal dynamics and particle}

\section{transport}

We next analyzed animals mutant for the gene eat-2, which encodes a non-alpha subunit of a nicotinic acetylcholine receptor (McKay et al., 2004). Because EAT-2 is

257 required for excitation of the pharynx by the motor neuron MC (Raizen et al., 1995)

258 animals mutant for eat-2 have a pump rate much lower than that of wild-type (1.3 \pm 0.5

$259 \mathrm{~Hz}$ compared to $4.4 \pm 0.48 \mathrm{~Hz}$ for N2). To assess differences in pump timing, we

260 normalized timing measurements by pump duration. After normalizing for pump

261 duration, we found that the duration of the contraction and relaxation phases were

262 nearly identical to that of N2. Consistent with previously reported results (Kozlova et al., 263 2019), we observed a higher rate of peristalsis in eat-2, with peristalsis occurring after

$26445.5 \%$ of pumps, compared to $22.6 \%$ in N2. Aside from slow pumping and increased 265 peristalsis, eat-2 mutants displayed normal pharyngeal muscle dynamics and particle 266 trapping (Fig. 5E, Video S8). Transport efficiency was similar to that of wild-type 267 worms, with about $65 \%$ of particles in the corpus becoming trapped in the isthmus.

\section{Discussion}

271 In this work we used high speed video microscopy to observe pharyngeal muscle

272 movements and particle transport in feeding mutants. We found that three of the four 
mutants described as slippery had defects in posteriorward transport, consistent with

274 previous observations (Avery, 1993a).

The mechanisms by which differences in pharyngeal movements create particle transport defects were clear only in a few cases. In eat-10, and possibly eat-15,

278 inefficiency of posterior transport could be explained by a deficiency or absence of anterior isthmus contraction. Isthmus contraction is required for particles to be drawn

280 from the metacorpus into the isthmus, prior to being trapped there.

Though pharyngeal muscles are electrically coupled (Starich et al., 1996) the pharynx exhibits highly compartmentalized muscle dynamics, between and even within individual muscle cells. For example, the anterior tips of the pm3 muscle cells, which span the procorpus, relax earlier than the rest of the corpus during pumping (Fang-Yen et al., 2009), a behavior that is antagonized by activity of the M1 neuron (Bhatla et al., 2015; Sando et al., 2021). Similarly, though the isthmus is composed of just one muscle cell type (pm5), the anterior and posterior compartments of the isthmus display different behaviors: the anterior isthmus contracts during pumps whereas the posterior isthmus displays a peristaltic movement after every fourth or five pumps. The finding of weak isthmus pumping but normal posterior isthmus peristalsis in eat-10 and possibly eat-15

292 mutants suggests that muscle contractions in subcellular compartments of the 293 pharyngeal isthmus are mediated by distinct mechanisms. 
295 In discussing reasons for isthmus peristalsis, Avery proposed that normal corpus and

296 terminal bulb function require them to be hydrodynamically isolated from each other

297 during pumping (Avery, 1993c). During corpus contraction, pharyngeal lumen pressure

298 decreases, and the bacteria suspension is drawn into the pharyngeal lumen from the

299 stoma. The posterior isthmus remaining closed (relaxed) at this time may serve to

300 prevent gastro-pharyngeal reflux, i.e. fluids and/or particles drawn into the pharynx from

301 the intestine. However, if the relaxed position of the posterior isthmus mechanically

302 isolates the terminal bulb from the corpus, then the timing of terminal bulb contraction

303 should not affect corpus to anterior isthmus transport. In all four slippery mutants

304 studied, we observed a premature terminal bulb relaxation (Fig 3). This observation

305 suggests that there is a connection between terminal bulb contraction and particle

306 transport efficiency, and that the TB is not fully isolated from the corpus and anterior

307 isthmus. It is possible that a delay between terminal bulb and corpus contraction

308 reduces the fluid resistance of the posterior pharynx, and so desynchronization of the

309 terminal bulb contraction relative to that of the corpus and isthmus normally helps to

310 maintain posteriorward flow.

312 We observed poor pharyngeal transport in animals mutant for eat-17, which encodes a

313 GTPase-activating protein required for pharyngeal cuticle structure (Straud et al., 2013).

314 The observation of poor particle transport despite no detectable defect in pharyngeal

315 motion timing suggests that the pharyngeal cuticle may play a role in transport, perhaps

316 via mechanical interactions with food particles. Alternatively, poorly macerated bacteria

317 in the terminal bulb due to eat-17 grinder dysfunction may affect the mechanics of 
318 bacterial transport in the entire pharynx, again perhaps because the terminal bulb is not

319 fully mechanically isolated from the corpus and anterior isthmus.

We found that eat- 6 and egl-19 mutants, previously described as defective in relaxation

(Avery 1993a), were most notable for their extended contraction phases. We also found

that both of these mutants were deficient in posteriorward particle transport. Therefore, these relaxation-defective mutants can also be described as slippery. Considering the

325 importance of the relaxation phase for trapping and transport, this result is not

326 surprising.

In this work we have focused on describing the behavioral defects in feeding mutants.

329 Future efforts will aim to connect the molecular identity of specific genes to specific

330 behavioral defects. The future use of genetically encoded calcium and/or voltage

331 sensors could allow for an assessment of excitation in different muscle compartments.

332 The combination of genetic analysis and high-resolution phenotyping of pharyngeal

333 behavior may lead to new insights into excitable cells and organs, in particular regarding

334 the mechanisms of subcellular compartmentalization of muscle contraction.

\section{Material and Methods}

338 Strains used in this study are shown in Figures 1 and 5. C. elegans were cultured 339 using standard methods (Stiernagle, 2006). For experiments, 5-10 young adult animals 340 were transferred to a $1 \mathrm{~mm}$ thick pad composed of $2 \%$ agarose in NGM buffer on a 
341 microscope slide, in addition to 1-2 $\mu \mathrm{L}$ of either an OP50 E. coli suspension in LB

342 medium or a $1 \%(\mathrm{vol} / \mathrm{vol})$ suspension of $1 \mu \mathrm{m}$ diameter polystyrene beads

343 (Polysciences 431), diluted in NGM buffer to $0.1 \%$ vol/vol. NGM buffer consisted of the

344 same components as NGM media (Stiernagle, 2006) without agar, peptone, or

345 cholesterol. The pad was covered with a \#1 cover slip and worms were given 15-45

346 minutes to acclimate to the pad before imaging. We mounted slides on an inverted

347 microscope (Nikon TE-2000S) with a 60X Plan Apo (NA 1.4) oil immersion objective

348 lens under differential interference contrast optics. For each animal we saved 1-3

349 recordings, each 4-5 s in duration, at 1000 fps using a Vision Research Phantom V9.1

350 high speed camera.

To record muscle timing data, we used a custom MATLAB script to review videos and manually recorded the frame numbers corresponding to 10 events: the start of corpus

354 contraction, the start of corpus relaxation, the end of corpus relaxation, the same for

355 both the isthmus and the terminal bulb, and the beginning of the next pump. This was

356 done only for recording periods in which all parts of the pump cycle were clearly visible.

357 We manually scored each pump for whether or not it was followed by isthmus

358 peristalsis.

360 To perform particle tracking, we used the same MATLAB program to analyze videos

361 frame by frame and recorded the approximate locations of several particles in each

362 pump, as well as the timings of each particle's beginning, peak, and end of movement.

363 To approximate particle locations, we established several guideposts along the pharynx 
364 (tip of the corpus, both ends of the metacorpus, the isthmus trap, the end of the anterior

365 isthmus, and the grinder) and assigned them approximate distances relative to the tip of

366 the stoma. We tracked at least one particle in the corpus and isthmus on every pump in

367 which there were particles in those compartments.

369 All statistical analyses were performed in Python. Because duration of components of

370 the pharyngeal pump is affected by the total pump time (Fang-Yen et al., 2009), we

371 normalized time measurements by the pump period for each trial before making

372 statistical comparisons between strains. To assess the significance of these particle

373 tracking samples, we used the 2x2 Fisher test, comparing each mutant strain with N2.

374 All pump timing data can be found in Supplemental Tables A1-3, and all particle

375 tracking data can be found in Supplemental Table A4.

\section{Acknowledgements}

378 Some strains were provided by the Caenorhabditis Genetics Center (CGC), which is 379 funded by NIH Office of Research Infrastructure Programs (P40 OD010440). We thank 380 Levi Kanu for conducting preliminary experiments in this project. 


\section{Video captions}

388 Video S1. High speed video of pharyngeal pumping in N2 animals. Pump cycles

389 from 4 wild type worms feeding on $1 \mu \mathrm{m}$ beads. The beginning of each animal's recording can be found at the following time points (m:s): Worm 1: 0:00; Worm 2: 0:13;

391 Worm 3: 0:27; Worm 4: 0:42. Scale bar $=25 \mu \mathrm{m}$ in all videos. Worm 1 corresponds to 392 tracking plot in Fig. 1b.

Video S2. High speed video of pharyngeal pumping in eat-10 animals. The

395 beginning of each animal's recording can be found at the following time points (m:s):

396 Worm 1: 0:00; Worm 2: 0:15; Worm 3: 0:30; Worm 4: 0:45. Note the weak or absent isthmus contractions in worms 3 and 4 . Worm 4 corresponds to tracking plot in Fig. 1c.

Video S3. High speed video of pharyngeal pumping in eat-13 animals. The

400 beginning of each animal's recording can be found at the following time points (m:s):

401 Worm 1: 0:00; Worm 2: 0:12; Worm 3: 0:28; Worm 4: 0:41. Note the deformed pharynx

402 in these worms. Worm 2 corresponds to tracking plot in Fig. 1d.

\section{3}

404 Video S4. High speed video of pharyngeal pumping in eat-15 animals. The

405 beginning of each animal's recording can be found at the following time points (m:s):

406 Worm 1: 0:00; Worm 2: 0:16; Worm 3: 0:29; Worm 4: 0:40. Worm 3 has weak isthmus

407 contraction. Worm 4 shows particles moving back and forth along the lumen during 
408 relaxation, perhaps due to the influence of the terminal bulb. Worm 2 corresponds to 409 tracking plot in Fig. 1e.

Video S5. High speed video of pharyngeal pumping in eat-17 animals. The beginning of each animal's recording can be found at the following time points (m:s): Worm 1: 0:00; Worm 2: 0:12; Worm 3: 0:27; Worm 4: 0:44. Note the deformed pharynxes in these worms. The grinder flaps are hard to resolve, indicating a potential defect in grinder formation. Worm 3 corresponds to tracking plot in Fig. 1f.

Video S6. High speed video of pharyngeal pumping in eat-6 animals. The beginning of each animal's recording can be found at the following time points (m:s): Worm 1: 0:00; Worm 2: 0:20; Worm 3: 0:48; Worm 4: 1:12. Note that the terminal bulb

420 does not come back to the fully relaxed position in these worms, but remains slightly 421 contracted even when the other compartments have closed. Worms 2-4 also have weak isthmus contraction. Worm 2 corresponds to tracking plot in Fig. 5c.

425 beginning of each animal's recording can be found at the following time points (m:s):

426 Worm 1: 0:00; Worm 2: 0:16; Worm 3: 0:40; Worm 4: 0:58. Note the unique "yawns" of

427 the terminal bulb in worms 1 and 2. In those pumps, the terminal bulb remained fully

428 contracted for two pump cycles. Worm 3 corresponds to tracking plot in Fig. $5 d$. 
430 Video S8. High speed video of pharyngeal pumping in eat-2 animals. The

431 beginning of each animal's recording can be found at the following time points (m:s):

432 Worm 1: 0:00; Worm 2: 0:27; Worm 3: 0:47; Worm 4: 1:15. Note the extended delay

433 between pump cycles in these worms compared to N2. Worm 2 corresponds to tracking

434 plot in Fig. 5e.

\section{References}

438

Avery, L. and Horvitz, H.R., 1987. A cell that dies during wild-type C. elegans development can function as a neuron in a ced-3 mutant. Cell, 51(6), pp.10711078.

Altun, Z.F., Hall, D.H., 2003. WormAtlas Hermaphrodite Handbook - Alimentary System - Pharynx. WormAtlas. https://doi.org/10.3908/wormatlas.1.3

Avery, L., 1993a. The genetics of feeding in Caenorhabditis elegans. Genetics 133, 897-917.

Avery, L., 1993b. Motor neuron M3 controls pharyngeal muscle relaxation timing in Caenorhabditis elegans. J. Exp. Biol. 175, 283-297.

Avery, L., 1993c. Motor neuron M3 controls pharyngeal muscle relaxation timing in Caenorhabditis elegans. J. Exp. Biol. 175, 283-297.

Bhatla, N., Droste, R., Sando, S.R., Huang, A., Horvitz, H.R., 2015. Distinct Neural Circuits Control Rhythm Inhibition and Spitting by the Myogenic Pharynx of C. elegans. Curr. Biol. CB 25, 2075-2089.

https://doi.org/10.1016/j.cub.2015.06.052 
453 Davis, M.W., Somerville, D., Lee, R.Y., Lockery, S., Avery, L., Fambrough, D.M., 1995. Mutations in the Caenorhabditis elegans Na,K-ATPase alpha-subunit gene, eat6, disrupt excitable cell function. J. Neurosci. Off. J. Soc. Neurosci. 15, 84088418.

Fang-Yen, C., Avery, L., Samuel, A.D.T., 2009. Two size-selective mechanisms specifically trap bacteria-sized food particles in Caenorhabditis elegans. Proc. Natl. Acad. Sci. U. S. A. 106, 20093-20096. https://doi.org/10.1073/pnas.0904036106

Kozlova, A.A., Lotfi, M., Okkema, P.G., 2019. Cross Talk with the GAR-3 Receptor Contributes to Feeding Defects in Caenorhabditis elegans eat-2 Mutants. Genetics 212, 231-243. https://doi.org/10.1534/genetics.119.302053 eat-18 are required for nicotinic neurotransmission in the Caenorhabditis elegans pharynx. Genetics 166, 161-169.

Raizen, D.M., Lee, R.Y., Avery, L., 1995. Interacting genes required for pharyngeal excitation by motor neuron MC in Caenorhabditis elegans. Genetics 141, 13651382.

Sando, S.R., Bhatla, N., Lee, E.L., Horvitz, H.R., 2021. An hourglass circuit motif transforms a motor program via subcellularly localized muscle calcium signaling and contraction. eLife 10, e59341. https://doi.org/10.7554/eLife.59341

Song, B., Avery, L., 2012. Serotonin activates overall feeding by activating two separate neural pathways in Caenorhabditis elegans. J. Neurosci. Off. J. Soc. Neurosci. 32, 1920-1931. https://doi.org/10.1523/JNEUROSCI.2064-11.2012 
476 Stiernagle, T., 2006. Maintenance of C. elegans. WormBook Online Rev. C Elegans

$477 \quad$ Biol. 1-11. https://doi.org/10.1895/wormbook.1.101.1

478 Straud, S., Lee, I., Song, B., Avery, L., You, Y.-J., 2013. The jaw of the worm: GTPase-

479 activating protein EAT-17 regulates grinder formation in Caenorhabditis elegans.

$480 \quad$ Genetics $195,115-125$. https://doi.org/10.1534/genetics.113.152538 